\title{
Apple SERRATE negatively mediates drought resistance by regulating MdMYB88 and MdMYB124 and microRNA biogenesis
}

Xuewei Li ${ }^{1}$, Pengxiang Chen ${ }^{1}$, Yinpeng Xie ${ }^{1}$, Yan Yan ${ }^{1}$, Liping Wang ${ }^{1}$, Huan Dang ${ }^{1}$, Jing Zhang ${ }^{1}$, Lingfei Xu', Fengwang $\mathrm{Ma}^{1}$ and Qingmei Guan ${ }^{1}$

\begin{abstract}
The function of serrate (SE) in miRNA biogenesis in Arabidopsis is well elucidated, whereas its role in plant drought resistance is largely unknown. In this study, we report that MdSE acts as a negative regulator of apple (Malus $X$ domestica) drought resistance by regulating the expression levels of MdMYB88 and MdMYB124 and miRNAs, including mdm-miR156, mdm-miR166, mdm-miR172, mdm-miR319, and mdm-miR399. MdSE interacts with MdMYB88 and MdMYB124, two positive regulators of apple drought resistance. MdSE decreases the transcript and protein levels of MdMYB88 and MdMYB124, which directly regulate the expression of MdNCED3, a key enzyme in abscisic acid (ABA) biosynthesis. Furthermore, MdSE is enriched in the same region of the MdNECD3 promoter where MdMYB88/ MdMYB124 binds. Consistently, MdSE RNAi transgenic plants are more sensitive to ABA-induced stomatal closure, whereas MdSE OE plants are less sensitive. In addition, under drought stress, MdSE is responsible for the biogenesis of mdm-miR399, a negative regulator of drought resistance, and negatively regulates miRNAs, including mdm-miR156, mdm-miR166, mdm-miR172, and mdm-miR319, which are positive regulators of drought resistance. Taken together, by revealing the negative role of MdSE, our results broaden our understanding of the apple drought response and provide a candidate gene for apple drought improvement through molecular breeding.
\end{abstract}

\section{Introduction}

Serrate (SE) in Arabidopsis is a conserved eukaryotic RNA processing factor that was first reported to mediate the formation of early juvenile leaves and phase length ${ }^{1}$. Encoding a $\mathrm{C}_{2} \mathrm{H}_{2}$ zinc-finger protein, $\mathrm{SE}$ is required for normal shoot development ${ }^{2}$. Moreover, SE influences the alternative splicing of pre-mRNAs that primarily affect the selection of alternative $5^{\prime}$ splice sites of first introns ${ }^{3}$. Other genes with alternative splicing affected by SE encode transcription factors, splicing factors, and stressrelated proteins ${ }^{3}$. SE also functions in intron splicing and the transcription of intronless genes by pausing and

\footnotetext{
Correspondence: Qingmei Guan (qguan@nwafu.edu.cn)

${ }^{1}$ State Key Laboratory of Crop Stress Biology for Arid Areas/Shaanxi Key Laboratory of Apple, College of Horticulture, Northwest A\&F University, Yangling, 712100 Shaanxi, PR China

These authors contributed equally: Xuewei Li, Pengxiang Chen
}

elongating polymerase II complexes to promote their association with these intronless target genes ${ }^{4,5}$. Moreover, label-free quantitative proteomic analysis has revealed that $\mathrm{SE}$ is regulated by abscisic acid (ABA) under flooding stress ${ }^{6}$. In addition to these functions, SE has a role in microRNA (miRNA) biogenesis ${ }^{7,8}$, and previous studies report that SE and Hyponastic Leaves1 (HYL1) form a complex with DICER1 to achieve efficient and precise processing of pri-miRNAs ${ }^{9}$.

Drought stress is a major limiting factor that affects the yield and quality of apple. Researchers have long sought to increase the drought resistance of apple trees using molecular tools, such as genetic transformation and QTL mapping of loci associated with water use efficiency ${ }^{10-14}$. To date, a number of genes have been reported to play positive or negative roles in apple drought resistance. For example, MdMYB88 and MdMYB124 are proven to be

\section{(c) The Author(s) 2020}

(c) (i) Open Access This article is licensed under a Creative Commons Attribution 4.0 International License, which permits use, sharing, adaptation, distribution and reproduction in any medium or format, as long as you give appropriate credit to the original author(s) and the source, provide a link to the Creative Commons license, and indicate if changes were made. The images or other third party material in this article are included in the article's Creative Commons license, unless indicated otherwise in a credit line to the material. If material is not included in the article's Creative Commons license and your intended use is not permitted by statutory regulation or exceeds the permitted use, you will need to obtain permission directly from the copyright holder. To view a copy of this license, visit http://creativecommons.org/licenses/by/4.0/. 
two positive regulators of apple drought stress that influence xylem formation and secondary cell wall deposition ${ }^{11}$. In addition, MdMYB88 and MdMYB124 bind to gene promoters containing the cis-element AACCG $^{11,13}$ to regulate expression.

miRNAs are $~ 20-25$ nucleotide (nt) endogenous small molecules involved in various plant processes, including development and environmental stresses ${ }^{15-17}$. For example, the overexpression of miR156 improves the drought tolerance of alfalfa (Medicago sativa) by silencing $S P L 13^{18}$, a squamosa promoter-binding-like protein that binds to a core GTAC sequence in the promoter region of dihydroflavonol-4-reductase $(D F R)$ to induce anthocyanin biosynthesis in response to drought stress ${ }^{18,19}$. miR393 is involved in the rice response to stress by targeting auxin receptors and plays negative roles in drought and salt stress $^{20}$. In addition, transgenic Arabidopsis plants overexpressing miR399 exhibit hypersensitivity to drought but enhanced tolerance to salt stress and exogenously applied $\mathrm{ABA}^{21}$. In the apple genome, 23 conserved, 10 less conserved, and 42 apple-specific miRNAs or families with distinct expression patterns have been identified; these miRNAs target various genes and represent a wide range of enzymatic and regulatory activities ${ }^{22}$. Genome-wide miRNA analysis has revealed that 61 and 35 miRNAs are differentially expressed in drought-tolerant and droughtsensitive apple hybrid progeny, respectively, under drought stress $^{23}$. Among these mdm-miRNAs, mdmmiR156 and mdm-miRn249 are two positive regulators of apple osmotic stress ${ }^{23}$.

ABA is a drought-induced phytohormone that plays important roles in plant responses to environmental stresses. Upon drought stress, ABA accumulates rapidly to promote stomatal closure and avoid water $\operatorname{loss}^{24,25}$. Exogenous ABA treatment effectively and sufficiently upregulates many stress-marker proteins in wheat and maize that are indicated to enhance drought tolerance $^{26,27}$. ABA also acts as a signaling molecule in response to drought stress. Rice (Oryza sativa) OsPM1 (PLASMA MEMBRANE PROTEIN1) encodes an ABA influx carrier that mediates the movement of ABA across the plasma membrane and plays important roles in drought responses ${ }^{28}$. Under drought conditions, elevated ABA induces the production of $\mathrm{H}_{2} \mathrm{O}_{2}$ in guard cells, and subsequent $\mathrm{H}_{2} \mathrm{O}_{2}$-activated $\mathrm{Ca}^{2+}$ channels mediate the influx of $\mathrm{Ca}^{2+}$ in intact guard cells to close stomata ${ }^{29,30}$.

In the current study, we provide evidence that MdSE participates in the drought resistance of apple by negatively regulating MdMYB88- and MdMYB124-mediated ABA homeostasis. MdSE also regulates the expression of miRNAs that play critical roles in drought resistance in apple. Our results highlight the roles of MdSE in the drought tolerance of apple and thereby provide genetic determinants for apple breeding.

\section{Results}

MdSE interacts with and reduces the transcript and protein levels of MdMYB88 and MdMYB124

When applying affinity-purified mass spectrometry analysis to ascertain the interacting partners of MdMYB88 and MdMYB124, the SERRATE protein, which usually participates in miRNA biogenesis, primiRNA, and pre-mRNA splicing in Arabidopsis, was identified $^{3}$. The interaction between MdSE and MdMYB88 or MdMYB124 was confirmed by BiFC analysis (Fig. 1a), which was further verified by a Co-IP assay (Fig. 1b). However, MdSE did not interact with MdMYB88 in yeast, as demonstrated by a yeast twohybrid analysis (Fig. S1), indicating that the physical interaction of MdSE with MdMYB88 or MdMYB124 may require another component.

The expression of MdSE was examined in MdMYB88 and MdMYB124 transgenic plants, which were generated previously $^{13}$. qRT-PCR analysis revealed no regulation of MdSE by MdMYB88 or MdMYB124 under control or dehydration conditions (Fig. S2). To assess whether $M d M Y B 88$ or $M d M Y B 124$ expression levels are regulated by MdSE, MdSE RNAi and MdSE OE plants were generated. The transgenic plants were verified at the DNA and RNA levels (Fig. S3). After air dehydration for $2 \mathrm{~h}$, transcripts of $M d M Y B 88$ or $M d M Y B 124$ were reduced dramatically in MdSE OE plants but increased in MdSE RNAi plants (Fig. 1c, d). Western blot analysis confirmed the downregulation of MdMYB88 and MdMYB124 by MdSE under drought (Fig. 1e), indicating that under drought conditions, MdSE decreases levels of MdMYB88 and MdMYB124 proteins.

Since SE is responsible for the alternative splicing of premRNAs in Arabidopsis, we then examined the transcripts of $M d M Y B 88$ and MdMYB124 in MdSE RNAi plants by a RT-PCR assay. We found that decreased MdSE levels did not affect splicing of $M d M Y B 88$ and MdMYB124 in apple under control or drought conditions (Fig. S4).

\section{MdSE subcellular localization and expression pattern}

Protein alignment demonstrated that MdSE shares 67.2\% sequence similarity with Arabidopsis SE and is more closely related to SERRATE from Prunus persica (Fig. S5). Based on a transient expression assay, the YFP-MdSE fusion protein was present in the nucleus of tobacco cells (Fig. 2a), consistent with the nuclear localization of SE in Arabidopsis $^{31}$. SE from M. prunifolia was found to be expressed predominantly in flowers, followed by stems, leaves, and roots (Fig. 2b). The MdSE expression level was reduced in response to drought stress (Fig. 2c).

\section{MdSE is a negative regulator of drought tolerance}

To understand the biological function of MdSE in the drought response of apple, the drought tolerance of 


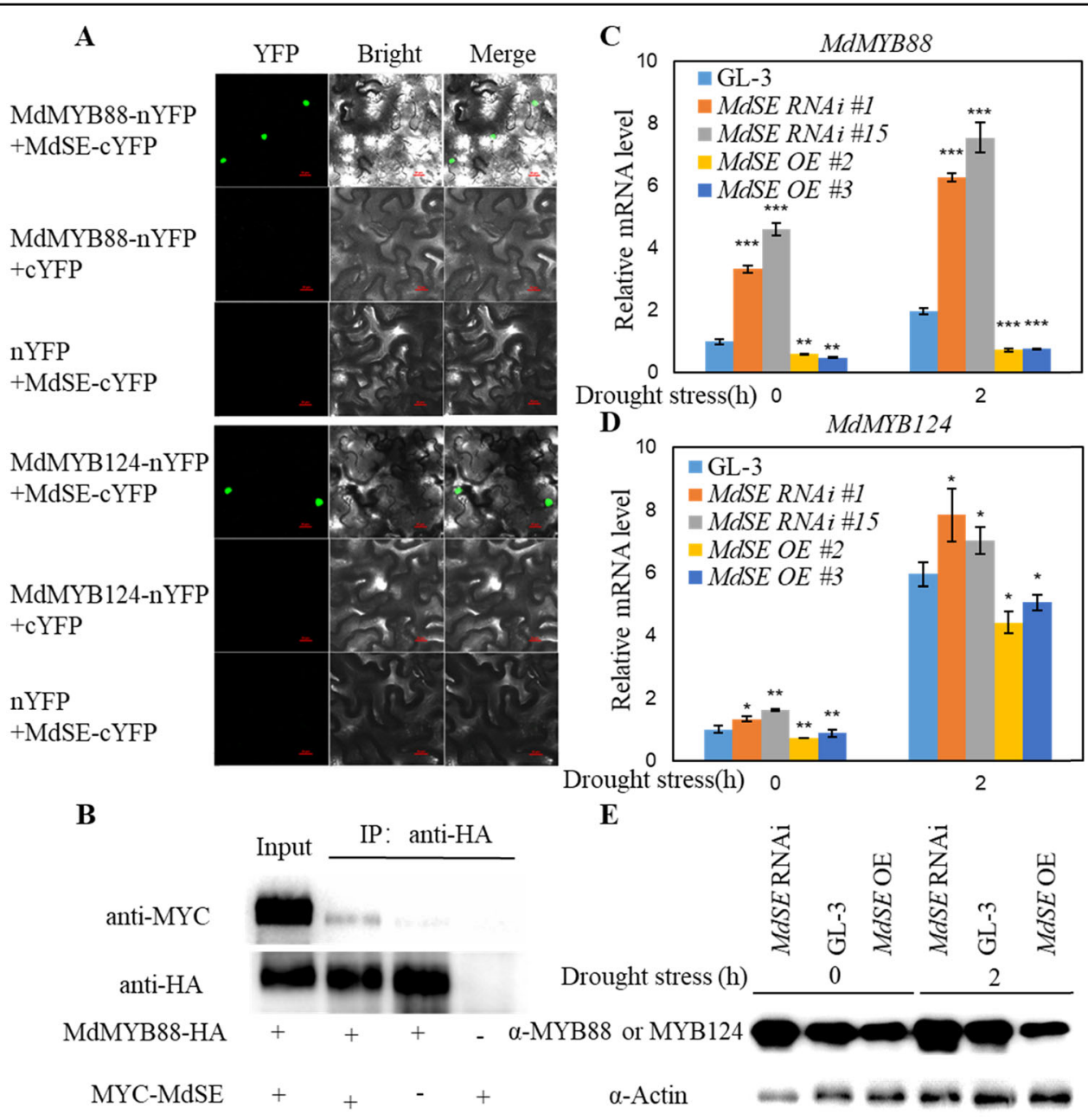

Fig. 1 MdSE reduces the transcript and protein levels of MdMYB88 and MdMYB124 by interacting with them. a MdSE interacts with MdMYB88 and MdMYB124 in tobacco leaves by BiFC analysis. Bars =20 $\mathrm{mm}$. b MdSE interacts with MdMYB88 in tobacco by Co-IP analysis. MdMYB88HA was coinfiltrated with MYC-MdSE in tobacco leaves. Proteins were extracted and immunoprecipitated with an anti-HA antibody. The immunocomplex was then detected with western blot using anti-MYC or anti-HA. c, d Expression level of MdMYB88 or MdMYB124 in GL-3, MdSE RNAi, and MdSE OE plants under control or drought conditions. Data are means \pm SD $(n=3)$. Student's $t$ test was performed, and statistically significant differences are indicated by ${ }^{*} P<0.05,{ }^{*} P<0.01$, or ${ }^{* * *} P<0.001$. e Protein level of MdMYB88 or MdMYB124 in GL-3, MdSE RNAi, and MdSE OE plants under control or drought conditions

5-month-old MdSE transgenic plants and nontransgenic GL-3 plants was examined. After withholding water for 30 days, and then rewatering for 7 days, $50-70 \%$ of $M d S E$ RNAi plants survived, whereas only $40 \%$ of the control plants were alive (Fig. 3a, b). Water loss from MdSE RNAi plants was much lower than loss from GL-3 plants (Fig. 3c). Compared with GL-3 plants under drought for 18 days, MdSE RNAi plants had higher photosynthesis rates and water use efficiency (Fig. 3d, e).

When exposed to drought for 24 days and rewatered for 7 days, $~ 30 \%$ of 5 -month-old MdSE OE plants survived; in contrast, $50 \%$ of GL-3 plants remained alive (Fig. 3f, g), which suggested that $M d S E$ OE plants are more sensitive to drought stress. In addition, $M d S E$ OE plants lost significantly more water under dehydration (Fig. 3h), and
MdSE OE plants had significantly lower photosynthesis rates and water use efficiency than GL-3 plants under drought (Fig. 3i, j). Together, these data suggest that $M d S E$ negatively regulates apple drought resistance.

\section{MdSE regulates stomatal aperture and ABA accumulation under drought}

Because MdSE interacts with MdMYB88 and MdMYB124 in vivo (Fig. 1) and MdMYB88 and MdMYB124 directly regulate 9-cis-epoxycarotenoid dioxygenase 3 (MdNCED3) expression and ABA content under drought conditions (unpublished), MdSE regulation of MdNCED3 transcripts and ABA content under drought was investigated. According to qRT-PCR analysis, drought-induced MdNCED3 expression was 


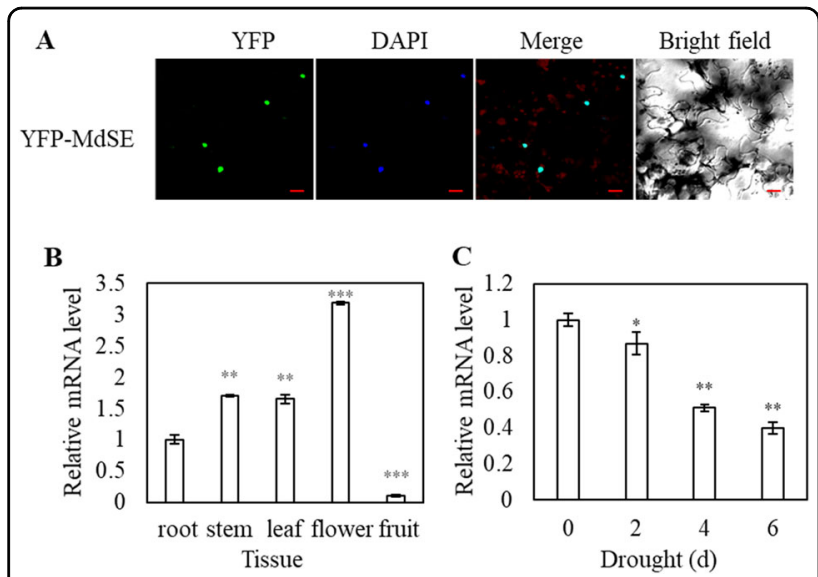

Fig. 2 MdSE localization and expression patterns. a MdSE is localized in the nucleus. Bars $=20 \mu \mathrm{m}$. $\mathbf{b}$ Expression of MdSE in different organs in M. prunifolia. c Transcript level of MdSE in response to drought. Error bars indicate the standard deviation $(n=3$ in $\mathbf{b}$ and c). Student's $t$ test was performed, and statistically significant differences are indicated by ${ }^{*} P<0.05$, ${ }^{*} P<0.01$, or ${ }^{*}{ }^{*} P<0.001$

significantly higher in MdSE RNAi plants but lower in $M d S E$ OE plants than in GL-3 plants (Fig. 4a). The ABA content was then measured in MdSE transgenic and GL-3 plants under control and drought conditions. LC-MS analysis showed that MdSE RNAi plants contained significantly more ABA but that $M d S E$ OE plants contained less $A B A$ in response to drought than GL-3 plants (Fig. 4b). Consistently, MdSE RNAi plants were hypersensitive to ABA-induced stomatal closure, whereas MdSE OE plants were less sensitive (Fig. $4 \mathrm{c}-\mathrm{f}$ ).

\section{MdSE is enriched in the MdNCED3 promoter}

Regulation of the main biosynthetic pathway of $\mathrm{ABA}$ is mediated by NCED3, which cleaves 9-cis-epoxycarotenoids and produces in the active isomer of $\mathrm{ABA}^{32}$. Because the ABA content of $M d S E$ transgenic lines was affected under drought conditions, we investigated whether $M d S E$ influences the $M d N E C D 3$ expression level by associating with the $M d N C E D 3$ promoter. Chromatin immunoprecipitation (ChIP) experiments were carried out using an SE-specific antibody, followed by qRT-PCR with the same primers used to assess MdMYB88 and MdMYB124 binding activity to MdNCED3. The ChIPqPCR results showed enrichment of MdSE at the $M d N C E D 3$ promoter in the same region where MdMYB88 and MdMYB124 bind (Fig. 5a, b). A dual luciferase reporter assay was also used to detect the influence of MdSE on MdNCED3 expression. The results showed that MdMYB88 and MdMYB124 enhanced $M d N C E D 3$ expression under both normal and dehydration conditions, whereas MdSE reduced the expression of $M d N C E D 3$. When MdSE was present, the expression of $M d N C E D 3$ induced by MdMYB88 or MdMYB124 was attenuated under control and drought conditions (Fig. $5 \mathrm{c}-\mathrm{f}$ ). These results suggest that the regulation of $M d N C E D 3$ by MdSE depends on its association with MdMYB88 and MdMYB124.

\section{MdSE regulates the biogenesis of miRNAs in apple under drought}

Arabidopsis SE is required for the biogenesis of miRNA $^{33,34}$. Loss-of-function mutant plants of SE have upward curling and radialized leaves ${ }^{35}$; secondary inflorescences lack an associated cauline leaf, and inflorescences often produce siliques that emerge from the same node on the stem ${ }^{34}$. Curly leaves on MdSE RNAi plants were not observed (Fig. 3), which may be due to $\sim 30-50 \%$ MdSE being maintained in MdSE RNAi plants (Fig. S3A). To understand whether MdSE has a similar function to Arabidopsis SE in miRNA biogenesis, we analyzed the expression of drought-responsive miRNAs, including mdm-miR156, mdm-miR166, mdm-miR172, mdm-miR319, and mdm-miR399, using stem-loop qPCR. Mdm-miR156, miR166, miR172, and miR319 are positive regulators of apple osmotic stress ${ }^{36}$ and drought resistance in alfalfa $^{18}$, Arabidopsis ${ }^{37,38}$, and creeping bentgrass $^{39}$, though miR399 is a negative regulator of drought resistance $^{21}$. The expression levels of these miRNAs were reduced in MdSE RNAi plants under control conditions (Fig. 6), suggesting a similar role for MdSE and SE in miRNA biogenesis. Under drought stress, compared with GL-3 plants, the expression levels of mdm-miR156, mdmmiR166, mdm-miR172, and mdm-miR319 increased in MdSE RNAi plants, whereas mdm-miR399 expression was reduced (Fig. 6), consistent with the drought tolerance phenotype of MdSE RNAi plants. These data indicate that $M d S E$ has a similar function in miRNA biogenesis as Arabidopsis SE and negatively modulates drought through regulation of drought-responsible miRNAs.

\section{Discussion}

In this study, we characterized a protein interacting with MdMYB88 and MdMYB124, MdSE, in response to drought. MdSE plays a negative role in apple drought resistance by negatively modulating the expression of MdMYB88 and MdMYB124, leading to the downregulation of MdNCED3 and reduced ABA levels. Furthermore, MdSE regulates the expression of droughtresponsive miRNAs under drought stress, which may contribute to its negative role in drought resistance.

The interaction between MdSE and MdMYB88 or MdMYB124 was confirmed by BiFC and Co-IP analyses. However, an in vitro interaction was not verified by $\mathrm{Y} 2 \mathrm{H}$ analysis, indicating the possibility of a bridge between MdSE and MdMYB88. For example, the interaction of Arabidopsis C-terminal domain phosphatase-like 1 

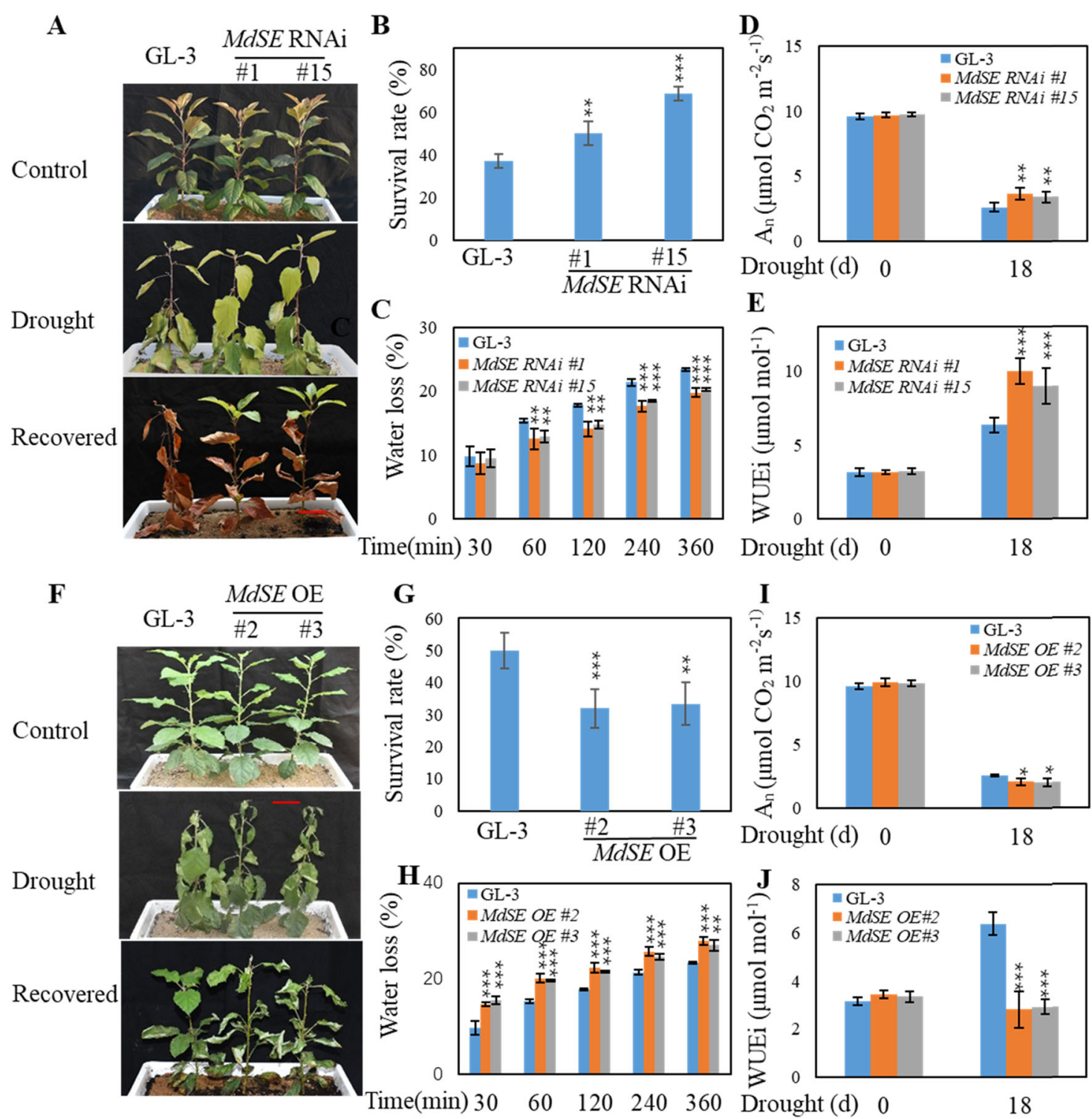

Fig. 3 MdSE transgenic plant response to drought stress. a, f Drought tolerance of GL-3 and transgenic plants. Bars $=5 \mathrm{~cm}$. Five-month-old MdSE $\mathrm{OE}$ and GL-3 plants were treated with drought for 24 days, and then rewatered for 7 days. MdSE RNAi and GL-3 plants were treated with drought for 30 days, and then rewatered for 7 days. $\mathbf{b}, \mathbf{g}$ Survival rate of GL-3 and transgenic plants shown in $\mathbf{a}$ and $\mathbf{f}$. Data are means $\pm \mathrm{SD}(n=36)$. $\mathbf{c}, \mathbf{h}$ Water loss of GL-3 and MdSE OE or RNAi plants under dehydration conditions for up to $360 \mathrm{~min}$. Data are means \pm SD $(n=10)$. $\mathbf{d}, \mathbf{i}$ The rate of photosynthesis $\left(A_{N}\right)$. e, $\mathbf{j}$ Intrinsic water use efficiency (WUEi). Data are means \pm SD $(n=15)$. Student's $t$ test was performed, and statistically significant differences are indicated by ${ }^{*} P<0.05,{ }^{*} P<0.01$, or ${ }^{* * *} P<0.001$

(CPL1) and HYL1 requires SE as a bridge ${ }^{31}$. MdHYL1 is a putative candidate for bridging MdSE and MdMYB88 or MdMYB124. Indeed, we found that MdHYL1 interacts with MdSE in $\mathrm{Y} 2 \mathrm{H}$ analysis (Fig. S6). The interaction between SE and HYL1 was also observed in Arabidopsis ${ }^{33}$, indicating that the functions of SE and HYL1 in some plant processes are conserved among plant species. Factors other than MdHYL1 might act as a bridge between MdSE and MdMYB88 or MdMYB124, but further study is necessary.
SE is responsible for alternative splicing of premRNAs ${ }^{3}$. In addition to the regulation of MdMYB88 and MdMYB124 transcripts by MdSE (Fig. 1), we hypothesized that MdSE might modulate the alternative splicing of $M d M Y B 88$ and MdMYB124. However, RT-PCR results showed that reduced $M d S E$ levels did not affect splicing of MdMYB88 and MdMYB124 in apple under control and drought conditions (Fig. S4). It is possible that MdSE can affect alternative splicing of other genes under control or drought conditions. The only challenge of 
A

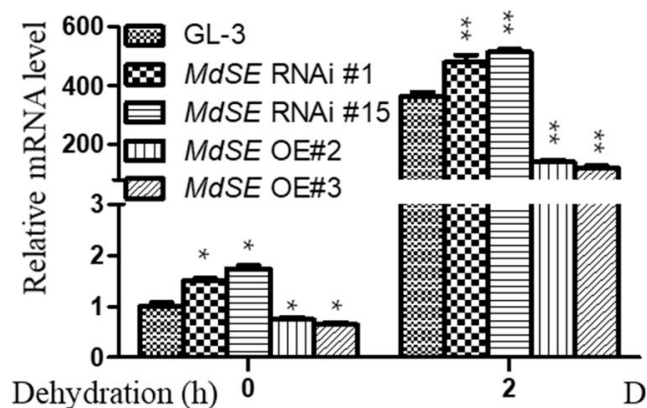

B

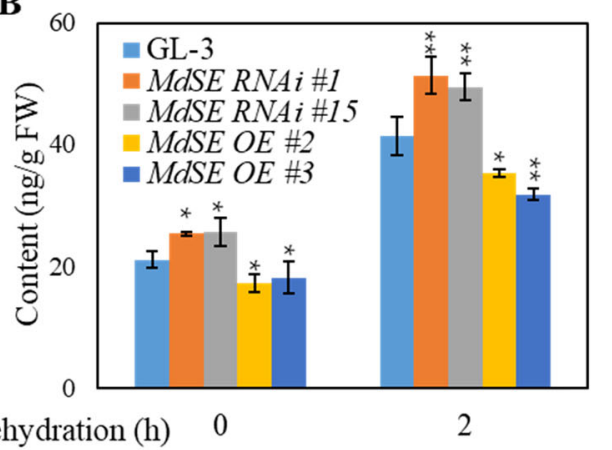

D

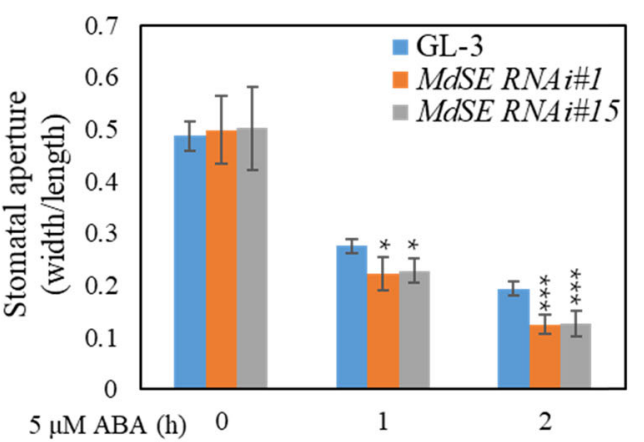

F

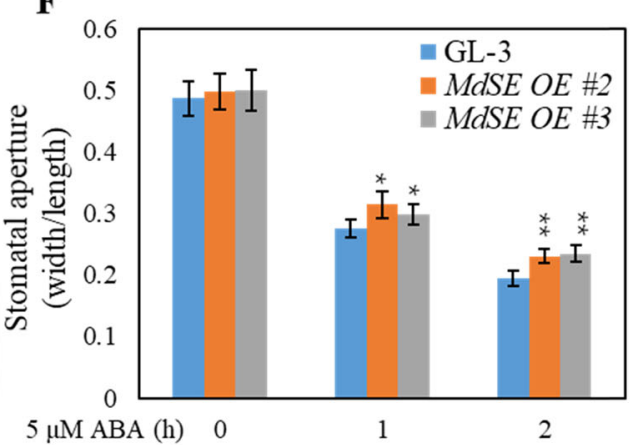

Fig. 4 ABA response and content in MdSE RNAi and MdSE OE plants. a Expression of MdNCED3 in GL-3, MdSE RNAi, or MdSE OE plants under control or dehydration conditions. Error bars indicate standard deviation $(n=3)$. Student's $t$ test was performed, and statistically significant differences are indicated by ${ }^{*} P<0.05$ or ${ }^{*} P<0.01$. b The ABA content in GL-3, MdSE RNAi, or MdSE OE plants under control or dehydration conditions. Error bars indicate standard deviation $(n=5)$. c, e Representative images of stomata of GL-3 and MdSE transgenic plants in response to ABA treatment. Bars $=10 \mu \mathrm{m}$. d, $\mathbf{f}$ Stomatal aperture of GL-3 and MdSE transgenic plants under ABA treatment. Data are the means \pm SD; 5 leaves were used, and at least 80 stomatal apertures were measured for each treatment. Student's $t$ test was performed, and statistically significant differences are indicated by ${ }^{*} P<0.05$, ${ }^{* *} P<0.01$, or ${ }^{* * *} P<0.001$

alternative splicing detection in apple is that $30-50 \%$ of MdSE was still functional in MdSE RNAi plants (Fig. S3A), which might affect the accuracy of analyses.

We demonstrated that MdSE is a negative regulator of drought resistance. First, survival ability analysis suggested that MdSE RNAi plants were more tolerant to drought stress but that MdSE OE plants were more sensitive (Fig. 3). Second, under dehydration conditions, $M d S E$ OE plants lost water more quickly than MdSE RNAi plants (Fig. 3c, h). Third, under drought stress, the cell membranes of MdSE RNAi plants were less damaged, as indicated by ion leakage (Fig. 3). Fourth, MdSE negatively regulated $\mathrm{ABA}$ accumulation and some droughtpositive miRNAs (Figs. 4 and $6 \mathrm{a}-\mathrm{d}$ ). In addition, MdSE positively regulated drought-negative mdm-miR399. Fifth, Arabidopsis se-1 mutants were also more tolerant to drought stress (Fig. S7). All these data support that MdSE plays a negative role in apple drought resistance and that the role of SE under drought might be conserved among plant species. 
A
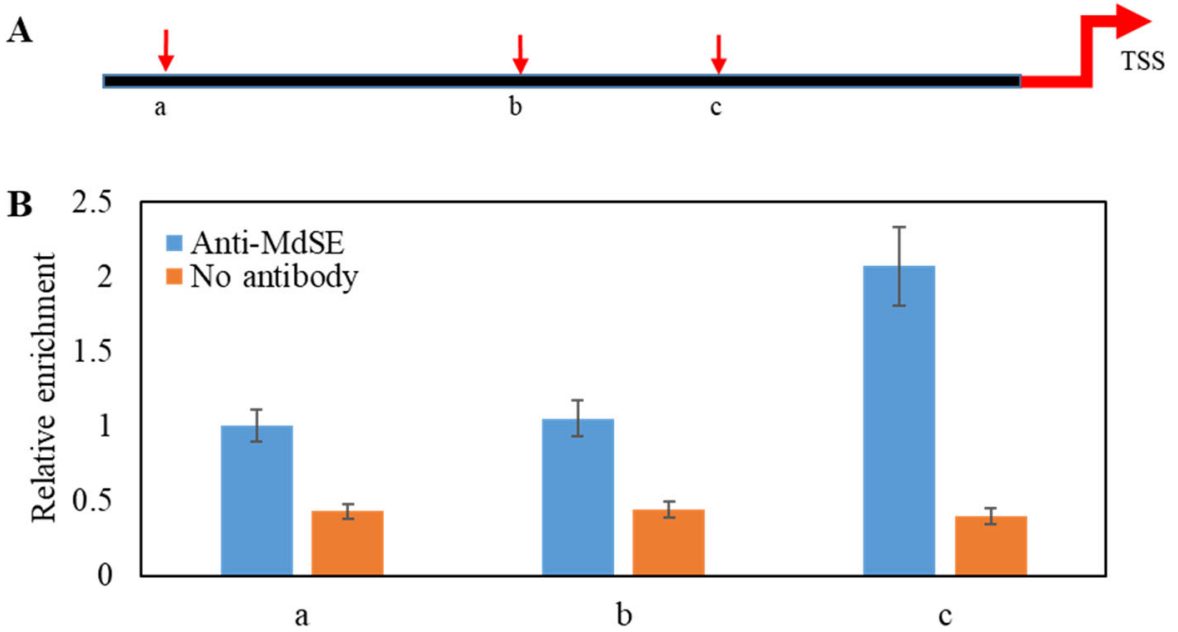

C

D
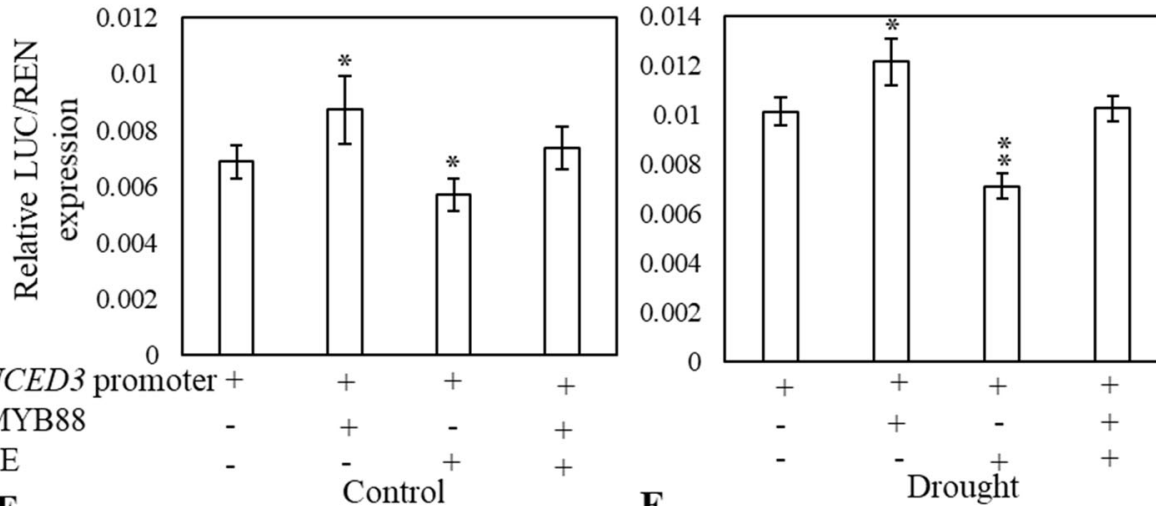

$\mathbf{E}$
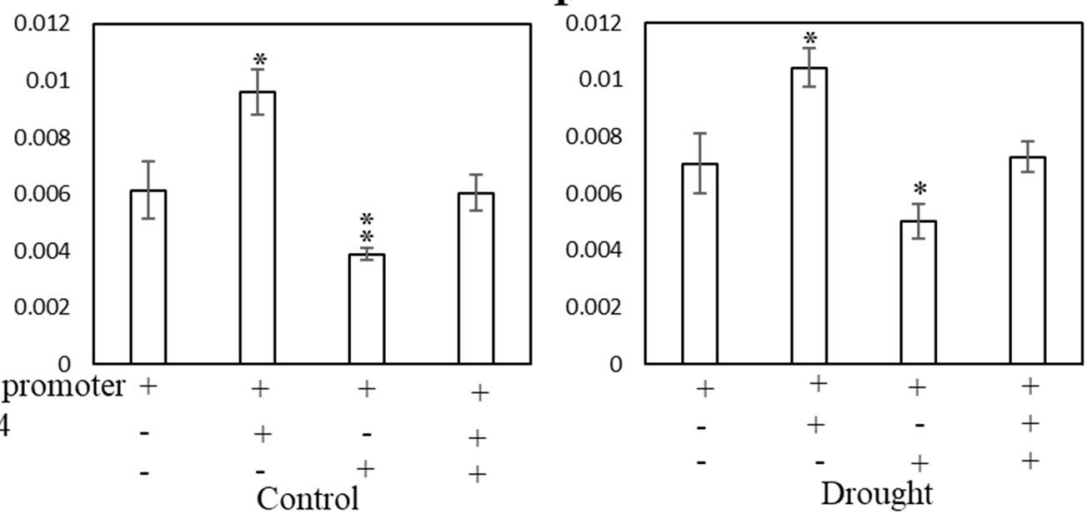

Fig. 5 MdSE is enriched in the MdNCED3 promoter and decreases MdNCED3 activity. a Diagram of MdNCED3 promoter regions. a-c Represent fragments containing two cis-elements of AGCCG from -1830 to $-1826 \mathrm{bp},-1368$ to $-1364 \mathrm{bp}$ and one cis-element of CGCGG from -880 to $-876 \mathrm{bp}$, respectively. For the negative control, no antibody was added. TSS transcription start site. b MdSE enrichment in the MdNCED3 promoter determined by ChIP-qPCR analysis. c-f Relative luciferase activity from dual luciferase reporter assays in N. benthamiana leaves. Pro35S::REN was used as an internal control. Quantification was performed by normalizing firefly luciferase activity to that of Renilla luciferase. Leaves of $N$. benthamiana were coinfiltrated and grown for $72 \mathrm{~h}$, and then leaves were collected or dehydrated for an additional $2 \mathrm{~h}$. Error bars indicate standard deviation $(n=10)$. Student's $t$ test was performed, and statistically significant differences are indicated by ${ }^{*} P<0.05$ or ${ }^{*} P<0.01$

$\mathrm{ABA}$ is a plant stress hormone that regulates stomatal closure within and outside guard cells through combinational mechanisms ${ }^{40}$. In Arabidopsis, maize, wheat, rice, and apple, elevated ABA content contribute to plant drought tolerance by inducing stomatal closure and stress-related signal transduction ${ }^{21,23,24,26,27,41,42}$. NCED3 is considered to be the key contributor to ABA production under water deficit conditions, and ZEP and AAO3 

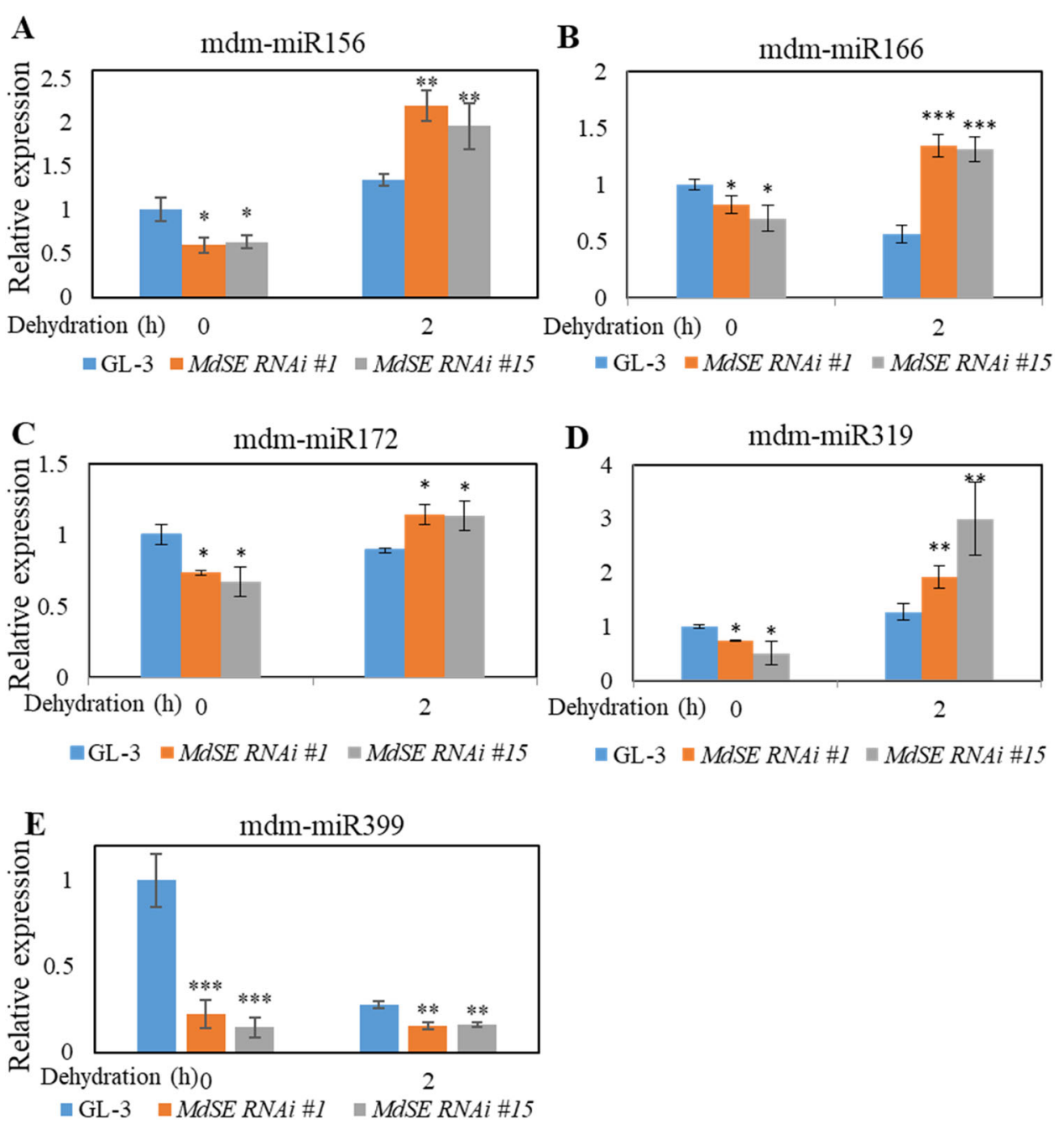

Fig. 6 MdSE affects the biogenesis of drought-responsive miRNAs in apple in response to drought. Mdm-miR156 (a), mdm-miR166 (b), mdmmiR172 (c), mdm-miR319 (d) and mdm-miR399 (e) were determined by stem-loop qPCR. Error bars indicate standard deviation $(n=3)$. Student's $t$ test was performed, and statistically significant differences are indicated by ${ }^{*} P<0.05$, ${ }^{*} P<0.01$, or ${ }^{* *} P<0.001$

play minor roles $^{43,44}$. In Arabidopsis, short vegetative phase (SVP) is able to bind to the promoters of the ABA catabolism pathway genes CYP707A1, CYP707A3, and $A t B G 1$, and thus contributes to ABA homeostasis ${ }^{45}$. Homeostasis of ABA was also regulated by reversible glycosylation mediated by ABA-UGTs (uridine diphosphate glucosyltransferases) to affect ABA bioactivity (Fig. S8) ${ }^{46,47}$. In our study, more ABA accumulated in $M d S E$ RNAi transgenic lines compared with MdSE OE and GL-3 plants (Fig. 4b). Such negative regulation of ABA content should contribute to the negative role of MdSE in drought resistance. We also found that MdSE was enriched at the promoter region of $M d N C E D 3$, which is the same region bound by MdMYB88 and MdMYB124 (Fig. 5). Considering the in vivo interaction between MdSE and MdMYB88 or MdMYB124, we conclude that the enrichment of MdSE at the MdNCED3 promoter was due to recruitment by MdMYB88 or MdMYB124 instead of direct binding.

Arabidopsis SE is a critical component required for primiRNA processing and miRNA biogenesis ${ }^{33,34}$. The results of microarray analysis showed that numerous miRNAs and their target genes are misexpressed in $s e-1$, including miR156, miR165, miR167, miR163, miR164, miR168, and miR171 ${ }^{5,33}$. In our study, stem-loop qPCR analysis demonstrated reduced levels of mdm-miR156, mdm-miR166, mdm-miR172, mdm-miR319, mdmmiR399, and mdm-miR398 transcripts in MdSE RNAi transgenic lines under normal environmental conditions (Fig. 6), indicating a conserved role for SE in miRNA biogenesis among plant species.

miRNAs participate in various plant processes, including root development ${ }^{48}$, flowering time ${ }^{49}$, apical dominance $^{35}$, and plant architecture ${ }^{16}$, and are also associated 
with tolerance to environmental stresses, including salt ${ }^{50}$, drought $^{18,21}$, cold ${ }^{51}$, and bacterial infection ${ }^{36}$. The overexpression of mdm-miR156 in apple calli enhances osmotic stress $^{22}$ and drought stress tolerance in alfalfa

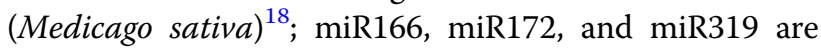
also reported to act as positive regulators of drought tolerance in rice ${ }^{38}$, soybean ${ }^{52}$, and creeping bentgrass (Agrostis stolonifera) $^{39}$, and miR399 plays a negative role in Arabidopsis drought resistance ${ }^{21}$. In our study, mdmmiR156, mdm-miR166, mdm-miR172, and mdm-miR319 were induced in MdSE RNAi transgenic plants after drought exposure, whereas mdm-miR399 was reduced, suggesting that these factors might contribute to the drought tolerance of MdSE RNAi plants.

In summary, our study elucidated the roles of MdSE in drought stress resistance. MdSE plays a negative role in drought resistance by affecting miRNA biogenesis and negatively regulating protein accumulation of MdMYB88 and MdMYB124, which results in negative regulation of ABA accumulation. Our study provides a deeper understanding of the complex mechanism of MdSE in response to drought stress and identifies a candidate gene for drought improvement through molecular breeding.

\section{Materials and methods}

\section{Plant materials and growth conditions}

For gene cloning, "Golden delicious" (Malus $\times$ domestica) grown in a greenhouse was used for RNA extraction. GL-3, a genotype selected from seedlings of "Royal Gala" (Malus $\times$ domestica), was used for genetic transformation $^{53}$. GL-3 grown on Murashige and Skoog (MS) medium ( $4.43 \mathrm{~g} / \mathrm{L}$ MS salts, $30 \mathrm{~g} / \mathrm{L}$ sucrose, and $7 \mathrm{~g} / \mathrm{L}$ agar, $\mathrm{pH}$ 5.8) supplemented with $0.2 \mathrm{mg} / \mathrm{L}$ 6-benzylaminopurine and $0.2 \mathrm{mg} / \mathrm{L}$ indoleacetic acid (IAA) under long-day conditions ( $14 \mathrm{~h} \mathrm{light} / 10 \mathrm{~h}$ dark cycle) for 4 weeks at $25^{\circ} \mathrm{C}$ were used for gene transformation. MdMYB88/124 RNAi plants and $M d M Y B 88$ or $M d M Y B 124$ overexpression plants were produced in a previous study ${ }^{13}$. The transgenic plants were rooted in MS medium (2.22 g/L MS salts, $20 \mathrm{~g} / \mathrm{L}$ sucrose, $7.5 \mathrm{~g} / \mathrm{L}$ agar, $0.5 \mathrm{mg} / \mathrm{L}$ IAA, $0.5 \mathrm{mg} / \mathrm{L}$ indolebutyric acid (IBA), pH 5.8) for 2 months, and then transplanted to substrate (Pindstrup, Denmark). se-1 was obtained from ABRC.

\section{RNA extraction and qRT-PCR analysis}

Detailed methods for RNA extraction and qRT-PCR analysis are provided in ref. ${ }^{13}$. The primers used for qRTPCR analysis are listed in Supplementary Table 1.

\section{Generation of transgenic apple}

To generate a construct for $M d S E$ overexpression, the coding region (CDS) of MdSE was cloned into pGWB414 to produce MdSE-pGWB414. To knock down MdSE, a 292-bp fragment of $M d S E$ was introduced into
pK7WIWG2D, resulting in MdSE-pK7WIWG2D. Both plasmids were transformed into Agrobacterium strain EHA105. For genetic transformation, we used an Agrobacterium-mediated transformation method. Plant transformation was carried out according to Dai et al. ${ }^{53}$. Briefly, 4-week-old GL-3 leaves were cut into strips in liquid MS medium ( $4.43 \mathrm{~g} / \mathrm{L}$ MS salts and $30 \mathrm{~g} / \mathrm{L}$ sucrose, $\mathrm{pH} 5.2)$ with EHA105 $\left(\mathrm{OD}_{600}=0.6-0.9\right)$ carrying the relevant plasmid for $15 \mathrm{~min}$. Then, the leaf strips were transferred into maintenance medium $(4.43 \mathrm{~g} / \mathrm{L} \mathrm{MS}+2 \mathrm{mg} / \mathrm{L}$ TDZ $+0.5 \mathrm{mg} / \mathrm{L} \quad \mathrm{NAA}+100 \mu \mathrm{M}$ acetosyringone $+1 \mathrm{mM}$ betaine $+7.5 \mathrm{~g} / \mathrm{L}$ agar $+30 \mathrm{mg} / \mathrm{L}$ sugar, $\mathrm{pH}=5.8$ ). After 3 days, leaf strips were transferred into selection medium $(4.43 \mathrm{~g} / \mathrm{L} \mathrm{MS}+2 \mathrm{mg} / \mathrm{L} \mathrm{TDZ}+0.5 \mathrm{mg} / \mathrm{L} \mathrm{NAA}+250 \mathrm{mg} / \mathrm{L}$ cefotaxime $+50 \mathrm{mg} / \mathrm{L}$ kanamycin $+7.5 \mathrm{~g} / \mathrm{L}$ agar $+30 \mathrm{mg} / \mathrm{L}$ sugar, $\mathrm{pH}=5.8$ ) for 4 weeks in the dark, and then incubated for 6 weeks under light. The transgenic buds that stayed green on selection medium were grown for $\sim 4$ weeks. DNA and RNA were extracted from the transgenic plants and GL-3 and used to detect transgene insertion and MdSE expression levels by PCR and RTqPCR, respectively. Transgenic plants with transgene insertion, as well as altered expression levels of MdSE, were selected for further experiments. The primers used are listed in Supplementary Table 1.

\section{Drought treatment}

Drought treatment was carried out by withholding water for a certain number of days, and then rewatering for 7 days, followed by calculation of the survival rate. Specifically, 5-month-old MdSE OE and GL-3 plants were treated with drought for 24 days, and then rewatered for 7 days. MdSE RNAi and GL-3 plants were treated with drought for 30 days, and then rewatered for 7 days. To obtain photosynthesis data (the rate of photosynthesis and intrinsic water use efficiency), a LiCor-6400 portable photosynthesis system (LiCor) was used.

Detached leaves from 5-month-old MdSE OE, MdSE RNAi, and GL-3 were used for the water loss assay.

\section{Stomatal aperture measurements}

For stomatal aperture measurements, we used leaves of 2-month-old soil-grown transgenic apple and GL-3 plants. Leaves were cut off and plunged into stomatal opening solution $\left(30 \mathrm{mM} \mathrm{KCl}, 0.1 \mathrm{mM} \mathrm{CaCl}_{2}\right.$, and $10 \mathrm{mM}$ MES-KOH, pH 6.15) under light $\left(120 \mu \mathrm{mol} \mathrm{m}^{-2} \mathrm{~s}^{-1}\right)$ for $2 \mathrm{~h}$ to induce stomatal opening as described ${ }^{54}$. Then, ABA was added to the stomatal opening solution to a final concentration of $5 \mu \mathrm{M}$. The leaf epidermis was observed for stomatal aperture with an EX30 microscope (SDPTOP) after ABA treatment for 1 or $2 \mathrm{~h}$. Stomatal length and width were measured by Image software, and stomatal aperture was then calculated. 


\section{Western blot}

Proteins were extracted from leaf samples with extraction buffer $(50 \mathrm{mM}$ Tris- $\mathrm{HCl}, \mathrm{pH} 7.5,150 \mathrm{mM} \mathrm{NaCl}$, $2 \mathrm{mM}$ EDTA, 10\% glycerol, 1\% NP-40, $1 \mathrm{mM}$ phenylmethylsulfonyl fluoride). Twenty micrograms of protein was separated by $10 \%$ SDS-PAGE and blotted onto PVDF membranes (Millipore) using standard methods. The blots were blocked for $2 \mathrm{~h}$ in PBS $\left(50 \mathrm{mM} \mathrm{Na}_{2} \mathrm{HPO}_{4}\right.$, $\mathrm{pH}$ 7.4) with $5 \%$ nonfat milk, after which anti-MdMYB88 and MdMYB124 or anti-actin (ABclonal, AC009) antibodies were added. After $2 \mathrm{~h}$, the blots were washed twice in PBS milk, and a secondary antibody (goat anti-rabbit horseradish peroxidase-conjugated, $1 \mathrm{mg} / \mathrm{mL}$; catalog no. HS101; Transgen Biotech) was added. After washing, the blots were treated with Bio-Rad ChemiDoc XRS + to visualize the signals.

\section{Yeast two-hybrid assay}

A yeast two-hybrid assay was carried out according to the manufacturer's manuals (Clontech, 630439, 630489). MdMYB88-155 aa and CDS of MdSE were introduced into pGBDT7. CDS of MdMYB88 or MdHYL1 was cloned into pGADT7. MdSE-pGBDT7 and MdMYB88-pGADT7 or MdHYL1-pGADT7 were cotransformed into yeast strain AH109. MdMYB88-155 aa-pGBDT7 and MdHYL1-pGADT7 were also cotransformed into yeast strain AH109. Positive clones were selected on SD/-LeuTrp, and then on SD/-Leu-Trp-His-Ade $+\mathrm{x}$ - $\alpha$-gal plates for the $\mathrm{x}$ - $\alpha$-gal assay. The primers used are listed in Supplementary Table 1.

\section{Subcellular localization, BiFC, and Co-IP assays}

To generate constructs for BiFC assays, we cloned the CDS of $M d M Y B 88$ and its paralog gene $M d M Y B 124$ into pSPYNE-35S; the CDS of MdSE was cloned into the pSPYCE-35S vector. For subcellular localization, the CDS of $M d S E$ was cloned into the pEearleyGate104 vector. Transient expression assays were performed according to Xie et al. $^{13}$. After 3 days, fluorescent signals in transformed tobacco leaves were then detected using a Nikon A1R/A1 confocal microscope (Nikon).

For Co-IP analysis, the CDS of $M d M Y B 88$ was amplified by PCR and cloned into pEarleyGate 101; the CDS of $M d S E$ was cloned into pEarleyGate 203. Co-IP analyses were performed as described previously ${ }^{55}$.

The primers used are listed in Supplementary Table 1.

\section{ChIP-qPCR}

ChIP-qPCR assays were performed as described previously ${ }^{13}$. Tissue-cultured GL-3 was used for crosslinking, and the ChIP assay was performed with an anti-SE antibody (Agrisera, AS09 532A). Three regions of the $M d N C E D 3$ promoter were examined by qPCR, with no antibody ChIP samples serving as the reference. The primers used for ChIP-qPCR are listed in Supplementary Table 1.

\section{ABA measurement}

ABA was extracted as described ${ }^{56}$. Frozen apple leaf samples (about $100 \mathrm{mg}$ fresh weight) was ground in liquid nitrogen, and then extracted with $1 \mathrm{ml}$ of cold extraction buffer (methanol:isopropanol:acetic acid $=20: 79: 1, \mathrm{v} / \mathrm{v} / \mathrm{v}$ ). After centrifugation at $4{ }^{\circ} \mathrm{C}$ and $12,000 \mathrm{rpm}$ for $10 \mathrm{~min}$, the supernatant was transferred into a $2 \mathrm{~mL}$ tube, and $500 \mu \mathrm{L}$ cold extraction buffer was added followed by vortexing for $5 \mathrm{~min}$. The extraction process was repeated three times followed by centrifugation at $4{ }^{\circ} \mathrm{C}$ and $12,000 \mathrm{rpm}$ for $10 \mathrm{~min}$. The supernatant was filtered through a $0.22 \mu \mathrm{m}$ PTFE filter (Waters, Milford, MA, USA). GC-MS analysis was carried out using a QTRAP 5500 LC-MS/MS (AB SCIEX, Redwood City, USA).

\section{Accession numbers}

Sequence data can be found in NCBI under the following numbers: MdMYB88 (KY569647), MdMYB124 (KY569648), MdSE (KY568649), and MdNCED3 (XM_008380174.2).

\section{Acknowledgements}

We thank Dr Zhihong Zhang from Shenyang Agricultural University for providing tissue-cultured GL-3 plants. This work was supported by the National Key Research and Development Program of China (2019YFD1000100) and the National Natural Science Foundation of China (31622049 and 31872080).

\section{Author contributions}

Q.G. designed the project. X.L., P.C., Y.Y., L.W., H.D., and J.Z. performed the experiments. Y.Y., L.W., H.D., Y.X., L.X., and F.M. analyzed the data. L.X. and P.C. wrote the manuscript.

\section{Conflict of interest}

The authors declare that they have no conflict of interest.

Supplementary Information accompanies this paper at (https://doi.org/ 10.1038/s41438-020-0320-6).

Received: 7 January 2020 Revised: 3 April 2020 Accepted: 6 April 2020 Published online: 01 July 2020

\footnotetext{
References

1. Clarke, J. H., Tack, D. F., Findlay, K. M., Van Montagu, M. \& Van Lijsebettens, M. The SERRATE locus controls the formation of the early juvenile leaves and phase length in Arabidopsis. Plant J. 20, 493-501 (1999).

2. Prigge, M. J. \& Wagner, D. R. The Arabidopsis SERRATE gene encodes a zincfinger protein required fornormal shoot development. Plant Cell 13, 1263-1279 (2001).

3. Raczynska, K. D. et al. The SERRATE protein is involved in alternative splicing in Arabidopsis thaliana. Nucleic Acids Res. 42, 1224-1244 (2014).

4. Christie, M. \& Carroll, B. J. SERRATE is required for intron suppression of RNA silencing in Arabidopsis. Plant Signal Behav. 6, 2035-2037 (2011).

5. Speth, $\mathrm{C}$. et al. Arabidopsis RNA processing factor SERRATE regulates the transcription of intronless genes. Elife 7, e37078 (2018).

6. Komatsu, S. et al. Label-free quantitative proteomic analysis of abscisic acid effect in early-stage soybean under flooding. J. Proteome Res. 1, 4769 (2013).
} 
7. Dong, Z., Han, M. H. \& Fedoroff, N. The RNA-binding proteins HYL1 and SE promote accurate in vitro processing of pri-miRNA by DCL1. Proc. Natl Acad. Sci. USA 105, 9970-9975 (2008).

8. Sascha, L. et al. Dual roles of the nuclear cap-binding complex and SERRATE in pre-mRNA splicing and microRNA processing in Arabidopsis thaliana. Proc. Natl Acad. Sci. USA 105, 8795-8800 (2008).

9. Sabin, L. R. et al. Ars2 regulates both miRNA- and siRNA- dependent silencing and suppresses RNA virus infection in Drosophila. Cell 138, 340-351 (2009).

10. An, J. P. et al. Apple MdMYC2 reduces aluminum stress tolerance by directly regulating MdERF3 gene. Plant Soil 418, 255-266 (2017).

11. Geng, D. L. et al. MdMYB88 and MdMYB124 enhance drought tolerance by modulating root vessels and cell walls in apple. Plant Physiol. 178, 1296-1309 (2018).

12. Sun, X. et al. Improvement of drought tolerance by overexpressing MdATG18a is mediated by modified antioxidant system and activated autophagy in transgenic apple. Plant Biotechnol. J. 16, 545-557 (2018).

13. Xie, Y. P. et al. An atypical R2R3 MYB transcription factor increases cold hardiness by CBF-dependent and CBF-independent pathways in apple. $N$. Phytol. 218, 201-218 (2018).

14. Wang, H. B. et al. Mapping QTLs for water-use efficiency reveals the potential candidate genes involved in regulating the trait in apple under drought stress. BMC Plant Biol. 18, 136 (2018).

15. Fischer, J. J., Beatty, P. H., Good, A. G. \& Muench, D. G. Manipulation of microRNA expression to improve nitrogen use efficiency. Plant Sci. 210, 70-81 (2013).

16. Jiao, Y. et al. Regulation of OsSPL14 by OsmiR156 defines ideal plant architecture in rice. Nat. Genet. 42, 541-544 (2010).

17. Zeng, Z., Liu, Z. \& Xia, R. Small RNAs, emerging regulators critical for the development of horticultural traits. Hortic. Res. 5, 63 (2018).

18. Arshad, M., Feyissa, B. A., Amyot, L., Aung, B. \& Hannoufa, A. MicroRNA156 improves drought stress tolerance in alfalfa (Medicago sativa) by silencing SPL13. Plant Sci. 258, 122-136 (2017)

19. Feyissa, B. A., Arshad, M., Gruber, M. Y., Kohalmi, S. E. \& Hannoufa, A. The interplay between miR156/SPL13 and DFRWD40-1 regulate drought tolerance in alfalfa. BMC Plant Biol. 19, 434 (2019).

20. Xia, K. F. et al. OsTIR1 and OsAFB2 downregulation via OsmiR393 overexpression leads to more tillers, early flowering and less tolerance to salt and drought in rice. PLoS ONE 7, e30039 (2012).

21. Baek, D. et al. A role for Arabidopsis miR399f in salt, drought, and ABA signaling. Mol. Cells 39, 111-118 (2016).

22. Xia, R., Hong, Z., An, Y. Q., Eric, P. B. \& Liu, Z. Apple miRNAs and tasiRNAs with novel regulatory networks. Genome Biol. 13, R47-R47 (2012).

23. Niu, C. et al. Genome-wide identification of drought-responsive microRNAs in two sets of Malus from interspecific hybrid progenies. Hortic. Res. 6, 75 (2019).

24. Lim, C. W., Baek, W., Jung, J., Kim, J. H. \& Lee, S. C. Function of ABA in stomatal defense against biotic and drought stresses. Int J. Mol. Sci. 16, 15251-15270 (2015).

25. Shinozaki, K. \& Yamaguchi-Shinozaki, K. Molecular responses to dehydration and low temperature: differences and cross-talk between two stress signaling pathways. Curr. Opin. Plant Biol. 3, 217-223 (2000).

26. Alvarez, S., Roy, C. S. \& Pandey, S. Comparative quantitative proteomics analysis of the ABA response of roots of drought-sensitive and drought-tolerant wheat varieties identifies proteomic signatures of drought adaptability. J. Proteome Res. 13, 1688-1701 (2014).

27. Zamora-Briseno, J. A. \& de Jimenez, E. S. A LEA 4 protein up-regulated by ABA is involved in drought response in maize roots. Mol. Biol. Rep. 43, 221-228 (2016).

28. Yao, L. et al. The AWPM-19 family protein OsPM1 mediates abscisic acid influx and drought response in rice. Plant Cell 30, 1258-1276 (2018).

29. Pei, Z. M. et al. Calcium channels activated by hydrogen peroxide mediate abscisic acid signalling in guard cells. Nature 406, 731-734 (2000).

30. Schroeder, J. I., Kwak, J. M. \& Allen, G. J. Guard cell abscisic acid signalling and engineering drought hardiness in plants. Nature 410, 327-330 (2001).

31. Manavella, P. A. et al. Fast-forward genetics identifies plant CPL phosphatases as regulators of miRNA processing factor HYL1. Cell 151, 859-870 (2012)

32. Schwartz, S. H., Tan, B. C., Gage, D. A., Zeevaart, J. A. \& Mccarty, D. R. Specific oxidative cleavage of carotenoids by VP14 of maize. Science $\mathbf{2 7 6}, \mathbf{1 8 7 2 - 1 8 7 4}$ (1997).
33. Lobbes, D., Rallapalli, G., Schmidt, D. D., Martin, C. \& Clarke, J. SERRATE: a new player on the plant microRNA scene. EMBO Rep. 7, 1052-1058 (2006).

34. Yang, L., Liu, Z., Lu, F., Dong, A. \& Huang, H. SERRATE is a novel nuclear regulator in primary microRNA processing in Arabidopsis. Plant J. 47, 841-850 (2010).

35. Grigg, S. P., Claudia, C., Angela, H. \& Miltos, T. SERRATE coordinates shoot meristem function and leaf axial patterning in Arabidopsis. Nature 437, 1022-1026 (2005)

36. Niu, D. et al. miRNA863-3p sequentially targets negative immune regulator ARLPKs and positive regulator SERRATE upon bacterial infection. Nat. Commun. 7, 11324 (2016).

37. Yang, T. et al. The interaction between miR160 and miR165/166 in the control of leaf development and drought tolerance in Arabidopsis. Sci. Rep. 9, 2832 (2019).

38. Zhang, J. et al. Knockdown of rice microRNA166 confers drought resistance by causing Leaf rolling and altering stem xylem development. Plant Physiol. 176, 2082 (2018).

39. Zhou, M. et al. Constitutive expression of a miR319 gene alters plant development and enhances salt and drought tolerance in transgenic creeping bentgrass. Plant Physiol. 161, 1375-1391 (2014).

40. Kuromori, T., Seo, M. \& Shinozaki, K. ABA transport and plant water stress responses. Trends Plant Sci. 23, 513-522 (2018).

41. Seiler, $C$. et al. ABA biosynthesis and degradation contributing to $A B A$ homeostasis during barley seed development under control and terminal drought-stress conditions. J. Exp. Bot. 62, 2615-2632 (2011).

42. Zhang, L. et al. Role of abscisic acid (ABA) in modulating the responses of two apple rootstocks to drought stress. Pak. J. Bot. 46, 117-126 (2014).

43. Endo, A. et al. Drought induction of Arabidopsis 9-cis-epoxycarotenoid dioxygenase occurs in vascular parenchyma cells. Plant Physiol. 147, 1984-1993 (2008).

44. Seo, M. et al. The Arabidopsis aldehyde oxidase 3 (AAO3) gene product catalyzes the final step in abscisic acid biosynthesis in leaves. Proc. Natl Acad. Sci. USA 97, 12908-12913 (2000).

45. Wang, Z. et al. The flowering repressor SVP confers drought resistance in Arabidopsis by regulating abscisic acid catabolism. Mol. Plant 11, 1184-1197 (2018).

46. Bowles, D., Isayenkova, J., Lim, E. K. \& Poppenberger, B. Glycosyltransferases: managers of small molecules. Curr. Opin. Plant Biol. 8, 254-263 (2005).

47. Yonekura-Sakakibara, K. \& Hanada, K. An evolutionary view of functional diversity in family 1 glycosyltransferases. Plant J. 66, 182-193 (2011).

48. Bazin, J., Bustos-Sanmamed, P., Hartmann, C., Lelandais-Briere, C. \& Crespi, M Complexity of miRNA-dependent regulation in root symbiosis. Philos. Trans. $R$. Soc. Lond. B 367, 1570-1579 (2012).

49. Gandikota, M. et al. The miRNA156/157 recognition element in the $3^{\prime}$ UTR of the Arabidopsis SBP box gene SPL3 prevents early flowering by translational inhibition in seedlings. Plant J. 49, 683-693 (2007).

50. Sun, $X$. et al. Identification of novel and salt-responsive miRNAs to explore miRNA-mediated regulatory network of salt stress response in radish (Raphanus sativus L.). BMC Genomics 16, 1-16 (2015).

51. Kis, A. et al. Polycistronic artificial miRNA-mediated resistance to wheat dwarf virus in barley is highly efficient at low temperature. Mol. Plant Pathol. 17 427-437 (2016).

52. Li, W., Wang, T., Zhang, Y. \& Li, Y. Overexpression of soybean miR172c confers tolerance to water deficit and salt stress, but increases ABA sensitivity in transgenic Arabidopsis thaliana. J. Exp. Bot. 67, 175 (2016).

53. Dai, $\mathrm{H}$. et al. Development of a seedling clone with high regeneration capacity and susceptibility to Agrobacterium in apple. Sci. Hortic. 164, 202-208 (2013)

54. Kwak, J. M. et al. Dominant negative guard cell $\mathrm{K}+$ channel mutants reduce inward-rectifying $\mathrm{K}+$ currents and light-induced stomatal opening in Arabidopsis. Plant Physiol. 127, 473-485 (2001).

55. Guan, Q. et al. The protein phosphatase RCF2 and its interacting partner NAC019 are critical for heat stress-responsive gene regulation and thermotolerance in Arabidopsis. Plant Cell 26, 438-453 (2014).

56. Müller, M. \& Munné-Bosch, S. Rapid and sensitive hormonal profiling of complex plant samples by liquid chromatography coupled to electrospray ionization tandem mass spectrometry. Plant Methods 7, 37 (2011). 